\title{
Large area APDs for low energy X-ray detection in intense magnetic fields
}

\author{
M. Boucher ${ }^{\mathrm{a}}$, O. Huot ${ }^{\mathrm{a}}$, P.E. Knowles ${ }^{\mathrm{a}}$, L. Ludhova ${ }^{\mathrm{a}}$, F. Mulhauser ${ }^{\mathrm{a}, *}$, \\ L.A. Schaller ${ }^{a}$, C.A.N. Conde ${ }^{b}$, J.M.F. Dos Santos ${ }^{b}$, L.M.P. Fernandes ${ }^{b}$, \\ J.F.C.A. Veloso ${ }^{\mathrm{b}}$, F. Kottmann ${ }^{\mathrm{c}}$, A. Antognini ${ }^{\mathrm{d}}$, R. Pohl ${ }^{\mathrm{d}}$, D. Taqqu ${ }^{\mathrm{e}}$ \\ ${ }^{a}$ Department of Physics, University of Fribourg, Chemin du Musée 3, CH-1700 Fribourg, Switzerland \\ ${ }^{\mathrm{b}}$ Department of Physics, University of Coimbra, Portugal \\ ${ }^{\mathrm{c}}$ ETHZ, Zürich, Switzerland \\ ${ }^{\mathrm{d}}$ Max-Planck-Institut für Quantenoptik, Garching, Germany \\ ${ }^{\mathrm{e}}$ Paul Scherrer Institute, Villigen, Switzerland
}

\begin{abstract}
An experiment to measure the energy difference between the $2 \mathrm{~S}-2 \mathrm{P}$ atomic levels (Lamb shift) in muonic hydrogen is being prepared at PSI. Since the energy levels of muonic hydrogen are a factor of 186 more energetic than those of hydrogen, according to the ratio of reduced masses, the transitions lie in the soft X-ray region. The experiment needs long-lived muonic hydrogen in the $2 \mathrm{~S}$ state. This is achieved by stopping a low energy muon beam in a small volume of low pressure hydrogen in a $5 \mathrm{~T}$ magnetic field. A pulsed beam from a tunable laser induces the $2 \mathrm{~S}-2 \mathrm{P}$ transition and the $1.9 \mathrm{keV}$ X-ray photons resulting from the $2 \mathrm{P}-1 \mathrm{~S}$ deexcitation will be detected. Measuring the coincidences between the laser pulse and the X-ray as a function of the laser wavelength allows us to determine the Lamb shift. In this presentation we will discuss the perspectives of using large area avalanche photodiodes for the direct detection of the Xrays. Compared to gaseous detectors, they are more compact and simpler in operation. They are also insensitive to magnetic fields.
\end{abstract}

(C) 2003 Elsevier Science B.V. All rights reserved.

PACS: 07.85.-m; 29.40.Wk; 85.60.Dw; 36.10.Dr

Keywords: Photon detectors; X-ray detection; Avalanche photodiodes; Magnetic field

\section{Introduction}

The Lamb shift spectroscopy in muonic hydrogen [1,2] requires a large area $2 \mathrm{keV} \mathrm{X}$-ray detector capable of operation in a $5 \mathrm{~T}$ magnetic

\footnotetext{
*Corresponding author.

E-mail address: francoise.mulhauser@unifr.ch (F. Mulhauser).
}

field. In the experiment, negative muons are stopped in a low-pressure hydrogen gas to form muonic hydrogen atoms in the $2 \mathrm{~S}$ metastable state. The muonic atoms are then subjected to a pulsed laser beam of $\sim 6 \mu \mathrm{m}$ wavelength, to induce the $2 \mathrm{~S} \rightarrow 2 \mathrm{P}$ transition. Once the muonic atom is in the $2 \mathrm{P}$ state, it decays immediately to the ground state with the emission of a $1.9 \mathrm{keV}$ photon. 
The 2S-2P energy difference can be determined by measuring as function of the laser wavelength the intensity of coincidences between the laser pulse and the delayed $1.9 \mathrm{keV}$ photon. A measurement of the Lamb shift in muonic hydrogen will allow us to determine the proton charge radius with a precision near $0.1 \%$. Its actual value remains uncertain, as discussed in detail in Refs. $[3,4]$.

To achieve the intended accuracy we need a detector with an energy resolution of better than $30 \%$ for $1.9 \mathrm{keV}$ X-rays. To differentiate between prompt X-rays, resulting from the $2 \mathrm{P}-1 \mathrm{~S}$ transition of the muonic cascade in hydrogen, from laser delayed events, a time resolution of better than $100 \mathrm{~ns}$ is also necessary. Because the number of muons stopping in the gas is fairly low, a large detection area will help to increase the final counting rate. One last important constraint is a detector capable of functioning in a $5 \mathrm{~T}$ magnetic field.

The potential of a xenon driftless gas proportional scintillation counter (GPSC) with a CsIcovered microstrip plate (MSP) in place of a photomultiplier tube has already been recognized $[5,6]$. The working prototypes were tested and gave excellent results. For technical reasons their scintillation high voltage was set on the entrance window. This setup cannot be used in the final experiment, due to the fact that negative muons will stop in the hydrogen gas located just above the detector entrance window, and the negative scintillation $\mathrm{HV}$ is sufficient to prevent the lowenergy muons from entering the $\mathrm{H}_{2}$ target region. Alternative designs are being worked upon, but suffer from (temporary) technical difficulties associated with the very restricted space available for the detector.

To overcome delays in the development of the above-described detectors, we consider using instead Large Area Avalanche Photodiodes (LAAPD) of $16 \mathrm{~mm}$ diameter ${ }^{1}$ as direct X-ray detectors [7]. In the following, we report now the behaviour of LAAPDs in an intense magnetic field.

\footnotetext{
${ }^{1}$ Advanced Photonix Inc., 1240 Avenida Acaso, Camarillo, CA 93012, USA.
}

\section{Experimental setup}

Avalanche photodiodes are compact, monolithic devices made of a silicon $\mathrm{p}-\mathrm{n}$ junction where the internal electric field can reach values high enough to permit electron multiplication by impact ionisation [8,9]. When a high voltage is applied to the photodiode only a small region of the p-layer located in the front part of the diode remains undepleted. The electric field is such that the maximum is located around the $\mathrm{p}-\mathrm{n}$ junction. An incident photon, absorbed in the p-depleted layer, produces electron-hole pairs which will be accelerated towards the $\mathrm{n}^{+}$-contacts. The acceleration of charges produces secondary electron-hole pairs which lead to gains of a few hundred.

LAAPDs of $16 \mathrm{~mm}$ diameter, biased at $1.8 \mathrm{kV}$, were used. The thickness of the drift region is about $10 \mu \mathrm{m}$, while the charge multiplication region begins at a depth of about $20 \mu \mathrm{m}$ inside the depleted region and has a thickness of around $20 \mu \mathrm{m}$.

Fig. 1 shows such a detector with radioactive sources. The LAAPD was irradiated by a ${ }^{54} \mathrm{Mn}$ or ${ }^{55} \mathrm{Fe}$ source, which produced X-rays at energy $5.41 \mathrm{keV}\left(\mathrm{Cr} \mathrm{K}_{\alpha}\right)$ and $5.90 \mathrm{keV}\left(\mathrm{Mn} \mathrm{K}_{\alpha}\right)$, respectively. The $\mathrm{X}$-rays interact in the silicon drift region, such that the resulting primary electrons are amplified by the intense electric field around the junction producing a pulse with an amplitude proportional to the X-ray energy.

Detector signals were fed through a low-noise charge preamplifier (Canberra, 2004) to a spectroscopy amplifier. A short shaping time constant of $100 \mathrm{~ns}$ was used to achieve the optimum energy resolution. The peak was fitted by a Gaussian function superimposed on a linear background. The centroid as well as the full-width at halfmaximum were determined.

The detectors were placed inside a $1 \mathrm{~m}$ long, $20 \mathrm{~cm}$ bore diameter superconducting solenoid, capable of achieving magnetic fields up to $5 \mathrm{~T}$, with a $10^{-4}$ uniformity over an axial distance of $30 \mathrm{~cm}$ from the centre [10,11]. The detector orientations inside the solenoid were chosen, as shown in Fig. 1, such that the LAAPD axis was either perpendicular to the magnetic field direction (the most unfavorable orientation) or parallel to it. 

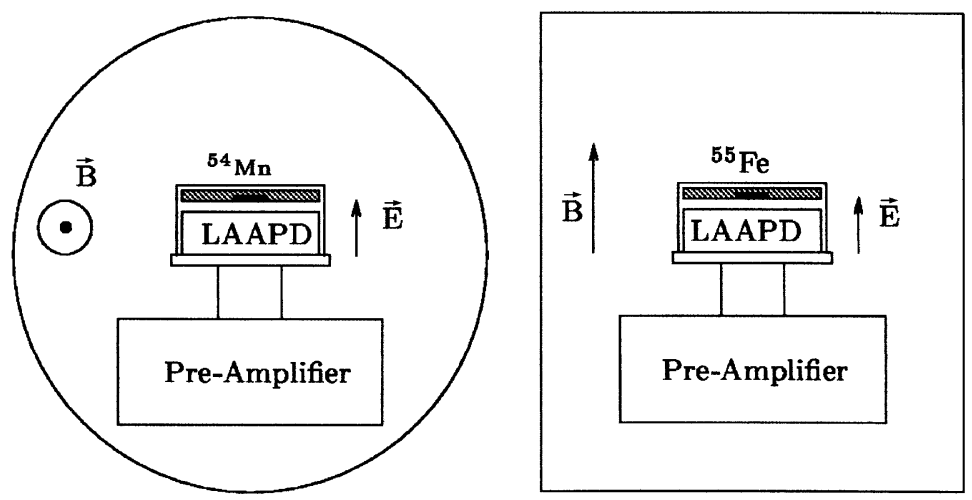

Fig. 1. Schematic of LAAPD orientations relative to the magnetic fields inside the solenoid. LAAPD, pre-amplifier, as well as sources are presented. The outside lines represent the solenoid bore. Dimensions are not to scale.
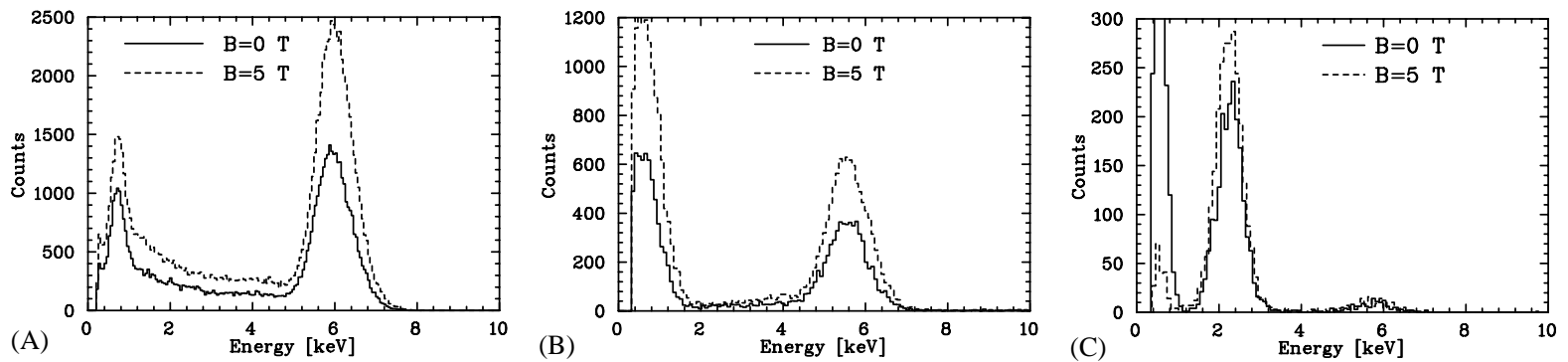

Fig. 2. Pulse-height distribution obtained for (A) $\mathrm{Mn} \mathrm{K}_{\alpha}$, (B) $\mathrm{Cr} \mathrm{K}_{\alpha}$, and (C) $\mathrm{S} \mathrm{K}_{\alpha}$ and a small intensity of Mn $\mathrm{K}_{\alpha} \mathrm{X}$-rays without and with a $5 \mathrm{~T}$ magnetic field.

\section{Experimental results and discussion}

Fig. 2 presents for three different X-ray sources energy spectra without a magnetic field as well as with a field of $5 \mathrm{~T}$. The third measurement was performed by irradiating a sulfur disk with a ${ }^{55} \mathrm{Fe}$ source. Actually, measurements were performed for all integer values between 0 and $5 \mathrm{~T}$. There was no significant degradation of the pulse height distribution. Measurement times were not the same, which explains the difference in statistics. It is important to notice that the orientation of the LAAPD relative to the magnetic field had no measurable effect.

The noise peak tail reaches $2 \mathrm{keV}$ for the single source measurements, whereas it was lower (around $1.5 \mathrm{keV}$ ) for the ${ }^{55} \mathrm{Fe}+\mathrm{S}$ tests. That was due to a better setup and less parasitic noise, which occurred when our setup was installed inside the solenoid.

Fig. 3 presents the relative pulse amplitude and energy resolution as a function of the magnetic field intensity for the three different sources. The magnetic field effects were small both for the amplitude and for the energy resolution. Amplitude variations of $2 \%$ were measured at 5.4 and $5.9 \mathrm{keV}$ and $5 \%$ at $2.3 \mathrm{keV}$, all within a $2 \sigma$ error. The energy resolutions, around $17 \%, 20.5 \%$, and $32 \%$, respectively, show variations within the measurement uncertainty.

The ${ }^{54} \mathrm{Mn}$ source was also used to measure timing resolution by setting the LAAPD in coincidence with a $\mathrm{NaI}$ detector. The $\mathrm{NaI}$ detected the $\gamma \mathrm{E} 2$ transition at $835 \mathrm{keV}$ and started the clock. When the LAAPD detected the $\mathrm{Cr} \mathrm{K}_{\alpha}$, the clock was stopped. By selecting coincidence events 

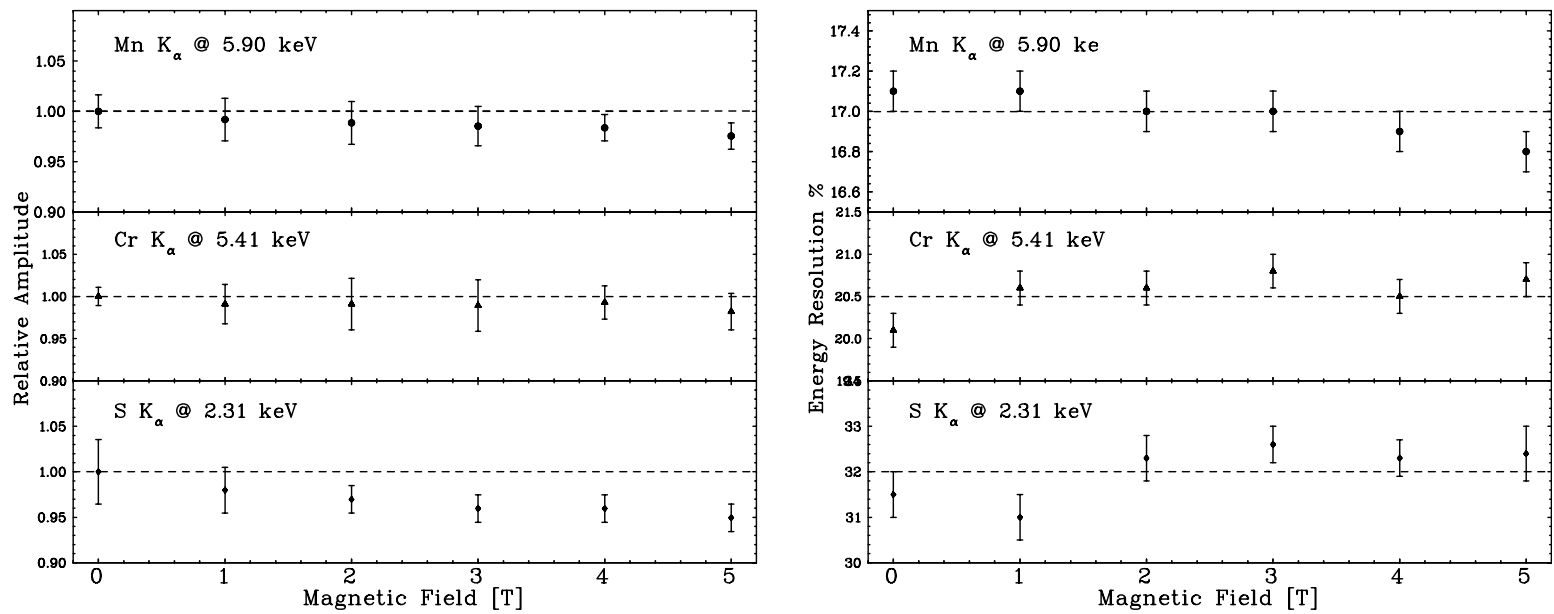

Fig. 3. Relative amplitude and energy resolution for $\mathrm{Mn} \mathrm{K}_{\alpha}, \mathrm{Cr} \mathrm{K}_{\alpha}$, and $\mathrm{S} \mathrm{K}_{\alpha}$ X-rays as a function of the magnetic field.

between the $\gamma E 2$ photopeak in $\mathrm{NaI}$ and the LAAPD $\mathrm{Cr} \mathrm{K}_{\alpha}$ peak, a time resolution of $12 \mathrm{~ns}$ (FWHM) for the two detectors was obtained. Knowing that $\mathrm{NaI}$ detectors have a time resolution of 3-5 ns, one can conclude that LAAPDs have a resolution of 10-12 ns. Unfortunately, this measurement was not performed as a function of the magnetic field, due to our NaI detector which was mechanically sensitive to the magnetic field. However, there is no reason to believe that the timing information will be dramatically changed, because we use the same signal for both energy and time.

In conclusion, we have shown that large area avalanche photodiodes can operate for X-ray detection in magnetic fields up to $5 \mathrm{~T}$ with negligible performance degradation. Additional measurements with visible and UV light were performed; visible light detection was B-field independant, while UV light detection had a strong B-field dependence [12]. The LAAPD detectors are therefore suitable for our experiment, matching all requested criteria. They will be used during the first muonic hydrogen Lamb shift experiment in summer 2002.

\section{Acknowledgements}

This work is supported by the Swiss National Science Foundation and the Portuguese Foundation for Science and Technology (FCT), project POCTI/FIS/13140/98.

\section{References}

[1] D. Taqqu, et al., Hyperfine Interactions 119 (1999) 311.

[2] F. Kottmann, et al., Hyperfine Interactions 138 (2001) 55.

[3] P.J. Mohr, B.N. Taylor, Rev. Mod. Phys. 72 (2000) 351.

[4] S.G. Karshenboim, Can. J. Phys. 77 (1999) 241.

[5] J.F.C.A. Veloso, et al., Nucl. Instr. and Meth. A 460 (2001) 297.

[6] J.F.C.A. Veloso, et al., Nucl. Instr. and Meth. A 489 (2002) 266

[7] L.M.P. Fernandes, J.A.M. Lopes, J.M.F. dos Santos, C.A.N. Conde, X-ray Spectrosc. 30 (2001) 164.

[8] A.Q.R. Baron, S.L. Ruby, Nucl. Instr. and Meth. A 343 (1994) 517

[9] E.M. Gullikson, E. Gramsch, M. Szawlowski, Appl. Opt. 34 (1995) 4662.

[10] D. Taqqu, Nucl. Instr. and Meth. A 247 (1986) 288.

[11] D. Taqqu, Z. Phys. C 56 (1992) s250.

[12] L.M.P. Fernandes, et al., Nucl. Instr. and Meth. A 498 (2002) 362 . 